\title{
Cinderella versus the Wicked Stepmother
}

\author{
Marijke Hans L. Bodlaender ${ }^{1}$, Cor A.J. Hurkens ${ }^{2}$, Vincent J.J. Kusters ${ }^{2}$, \\ Frank Staals ${ }^{2}$, Gerhard J. Woeginger ${ }^{2}$, and Hans Zantema ${ }^{2}$ \\ 1 Dept. of Information and Computing Sciences, Universiteit Utrecht, Netherlands \\ 2 Dept. of Mathematics and Computer Science, TU Eindhoven, Netherlands
}

\begin{abstract}
We investigate a combinatorial two-player game, in which one player wants to keep the behavior of an underlying water-bucket system stable whereas the other player wants to cause overflows. This game is motivated by data management applications in wireless sensor networks. We construct optimal strategies and characterize optimal bucket sizes for many instances of this game.
\end{abstract}

\section{Introduction}

Motivated by a data management application in wireless sensor networks, Bender \& al [1] study the minimum-backlog problem which is a two-player game on an undirected graph. The vertices of the graph contain buckets (which model buffers) that can store water (which models data). In every time step the adversary distributes exactly one liter of water over the buckets. The player then moves from his current vertex to an adjacent one and empties the corresponding bucket. The player's objective is to minimize the maximum amount of water in any bucket at any time, or in other words, to prevent the buckets from overflowing while using the smallest possible bucket size. Bodlaender \& al [2] discuss another variant where in every time step the player can empty a subset of buckets standing in an arbitrary independent set in the graph. Polishchuk \& Suomela [7] investigate the variant of the minimum-backlog problem where the underlying metric space is not a graph but the Euclidean plane. Chrobak \& al 3 ] discuss related scenarios in undirected graphs where data/water arrives continuously over time and where the player can (continuously) empty an entire independent set of buckets/buffers; if the player spends $t$ time units on a bucket set, then the contents of each such bucket is decreased by $t$. Note that in [1] the graph structure constrains the route taken by the player, whereas in [3] the graph structure constrains the sets of buckets that the player can empty simultaneously.

In the current paper we will concentrate on discrete scenarios where data/water arrives in rounds and where in every round the player can empty certain subsets of the buckets. One of the simplest cases of our game is as follows 86 .

"Five empty buckets of capacity b stand in the corners of a regular pentagon. Cinderella and her wicked Stepmother play a game that goes through a sequence of rounds: at the beginning of every round, the Stepmother takes one liter of water from the nearby river, and distributes it arbitrarily over the five buckets. 
Then Cinderella chooses a pair of neighboring buckets, empties them into the river, and puts them back into the pentagon. Then the next round begins. The Stepmother's goal is to make one of these buckets overflow. Cinderella's goal is to prevent this. For which bucket sizes b can the Stepmother eventually enforce a bucket overflow? And for which bucket sizes can Cinderella keep the game running forever?"

We study a general bucket game $\operatorname{BG}(n, c)$ for integers $n$ and $c$ with $1 \leq c \leq n-1$, where there are $n \geq 2$ buckets standing in a circle. Throughout we will use the term Cinderella to denote the player and the wicked Stepmother to denote the adversary. In every round the Stepmother first distributes one liter over the $n$ buckets, and then Cinderella empties an arbitrary group of $c$ consecutive buckets. The Stepmother wants to reach a bucket overflow, and Cinderella wants to avoid this. Clearly the above Cinderella puzzle coincides with BG(5,2). We define $F^{\prime}(n, c)$ as the infimum of all bucket sizes for which Cinderella can keep the game running forever, and we furthermore introduce the quantity $F(n, c)=$ $F^{\prime}(n, c)-1$. If Cinderella consistently avoids overflows for buckets of size $F^{\prime}(n, c)$, then at the end of every round she will only leave buckets with contents $F(n, c)$ or less.

Summary of Results. Table 1 lists the values $F(n, c)$ for all games with $n \leq 12$ buckets. For every single entry in this table with $n \leq 10$ we have proofs that were constructed by humans (and that are presented in this paper) as well as computerized proofs (that have been done with the SMT solver YICES [5]). For some of the entries in the lines $n=11$ and $n=12$, we only have computer proofs.

The entries in the table might seem somewhat chaotic at first sight. But taking a second look, the reader perhaps notices that the topmost numbers $1,1 / 2,1 / 3$, $\ldots, 1 / 11$ in the columns are the reciprocals of the positive integers. This indeed is a (fairly shallow) mathematical fact which we present in Section 3 , and which says that $F(c+1, c)=1 / c$ for all $c \geq 1$. Next let us discuss the values $F(c+2, c)$ immediately below the topmost numbers, which are

$$
3 / 2, \quad 1,5 / 9,1 / 2,7 / 20,1 / 3,9 / 35,1 / 4,11 / 54,1 / 5 .
$$

We note that the values in the even positions again are the reciprocals of integers. Indeed Section 2 shows that the function values $F(n, c)$ only depend on the ratio $n / c$, which for even $c=2 s$ implies $F(2 s+2,2 s)=F(s+1, s)=1 / s$. Section 3 shows that the remaining values in the odd positions satisfy $F(c+2, c)=(2 c+$ $4) /\left(c^{2}+3 c\right)$. By stepping further down in the columns, we meet the values $F(c+3, c)$ which read

$$
11 / 6, \quad 1, \quad 1,17 / 30,1 / 2,1 / 2,69 / 196,1 / 3,1 / 3 .
$$

The values $F(3 s+3,3 s)=F(s+1, s)=1 / s$ are of course once again the reciprocals of the integers. Section 3 shows that also the values $F(3 s+2,3 s-1)=$ $1 / s$ are such reciprocals, and it fully explains the (more complicated) structure of the remaining values $F(3 s+1,3 s-2)$. 
Table 1. Summary of the values $F(n, c)$ for $n \leq 12$ buckets. For the entries with $n \leq 10$, we even have proofs constructed by humans.

\begin{tabular}{|r|cccccccccc|}
\hline$n \backslash c$ & 1 & 2 & 3 & 4 & 5 & 6 & 7 & 8 & 9 & 10 \\
\hline 2 & 1 & - & - & - & - & - & - & - & - & - \\
3 & $3 / 2$ & $1 / 2$ & - & - & - & - & - & - & - & - \\
4 & $11 / 6$ & 1 & $1 / 3$ & - & - & - & - & - & - & - \\
5 & $25 / 12$ & 1 & $5 / 9$ & $1 / 4$ & - & - & - & - & - & - \\
6 & $137 / 60$ & $3 / 2$ & 1 & $1 / 2$ & $1 / 5$ & - & - & - & - & - \\
7 & $49 / 20$ & $3 / 2$ & 1 & $17 / 30$ & $7 / 20$ & $1 / 6$ & - & - & - & - \\
8 & $363 / 140$ & $11 / 6$ & 1 & 1 & $1 / 2$ & $1 / 3$ & $1 / 7$ & - & - & - \\
9 & $761 / 280$ & $11 / 6$ & $3 / 2$ & 1 & $299 / 525$ & $1 / 2$ & $9 / 35$ & $1 / 8$ & - & - \\
10 & $7129 / 2520$ & $25 / 12$ & $3 / 2$ & 1 & 1 & $5 / 9$ & $69 / 196$ & $1 / 4$ & $1 / 9$ & - \\
\hline 11 & $7381 / 2520$ & $25 / 12$ & $3 / 2$ & 1 & 1 & $77 / 135$ & $1 / 2$ & $1 / 3$ & $11 / 54$ & $1 / 10$ \\
12 & $83711 / 27720$ & $137 / 60$ & $11 / 6$ & $3 / 2$ & 1 & 1 & $5 / 9$ & $1 / 2$ & $1 / 3$ & $1 / 5$ \\
\hline
\end{tabular}

Moving further down in Table 1, we eventually hit an area that entirely consists of 1-entries. The uppermost 1-entry in every column is $F(2 c, c)=F(2,1)=$ 1 , and these entries form the so-called half-diagonal of the table (the diagonal where $c$ is half of $n$ ). This half-diagonal is a natural separation line, and it turns out that the combinatorics of the games below the half-diagonal behaves quite differently from the combinatorics of the games above the half-diagonal. Going even further down, we see that the lowermost 1-entry in every column is $F(3 c-1, c)=1$. In other words $F(n, c)=1$ holds whenever $2 c \leq n \leq 3 c-1$, and this is a mathematical theorem which we establish in Section 5. This theorem actually is our main result, and its proof is long and involved and uses quite delicate invariants.

What else is going on below the half-diagonal? The first column lists the harmonic numbers $H_{k}=1+\frac{1}{2}+\frac{1}{3}+\ldots+\frac{1}{k}$. The second column (below the halfdiagonal) seems to list again the harmonic numbers, but this time with every term occurring twice. And also the third column (below the half-diagonal) seems to list the harmonic numbers, with every term occurring thrice. And so on. We settle the behavior of the first column in Section 5, and we furthermore derive some partial results on the other columns. Many questions remain open.

Also the global structure of Table 1 shows many interesting properties. Of course, the values in every row form a non-increasing sequence (since $c$ increases and Cinderella becomes more powerful), and for similar reasons the values in 
every column form a non-decreasing sequence. In fact an even stronger property holds true (see Section 2): the function $F(n, c)$ is non-decreasing in the ratio $n / c$. Here is one application of this fact: from $F(3,2)=F(8,5)=1 / 2$ and from $3 / 2<11 / 7<8 / 5$ we immediately deduce $F(11,7)=1 / 2$.

Organization of the Paper. Section 2 states simple observations, summarizes the notation, and explains the general setup of our proofs. Section 3 deals with the games above the half-diagonal, and Section [5 deals with the games below the half-diagonal. Section 6 gives some conclusions.

\section{Preliminaries, Notations, and Conventions}

The $n$ buckets in any fixed game $\operatorname{BG}(n, c)$ are ordered along the circle and denoted $1,2, \ldots, n$. The numbering of buckets is always taken modulo $n$, so that $k$ and $n+k$ denote the same bucket. We use $d(i, j)=\min \{|i-j|, n-|i-j|\}$ to denote the distance between buckets $i$ and $j$ along the circle. If $d(i, j) \geq c$, then Cinderella can not simultaneously empty $i$ and $j$ within a single round. A subset $S$ of buckets is called independent, if it does not contain two adjacent buckets. The family $\mathcal{I}$ consists of all independent bucket subsets.

The contents of the buckets at a particular moment in time are often summarized in a vector $x=\left(x_{1}, \ldots, x_{n}\right)$ where $x_{i}$ denotes the current contents of bucket $i$. For a subset $S$ of the buckets, we use $x(S)=\sum_{i \in S} x_{i}$. To keep the notation simple, we write $x(i, j)$ short for $x(\{i, j\})$ and $x(i, j, k)$ short for $x(\{i, j, k\})$, and we use $x_{i}$ and $x(i)$ interchangeably.

The following two lemmas imply that function $F(n, c)$ only depends on the ratio $n / c$, and that it is non-decreasing in this ratio.

Lemma 1. $F(\lambda n, \lambda c)=F(n, c)$ for all integers $\lambda \geq 1$.

Proof. Consider an arbitrary strategy for the Stepmother for BG $(n, c)$. The Stepmother can emulate this strategy in $\mathrm{BG}(\lambda n, \lambda c)$ by using the buckets $\lambda, 2 \lambda, \ldots, n \lambda$. This yields $F(\lambda n, \lambda c) \geq F(n, c)$. Vice versa, Cinderella can carry over strategies from $\operatorname{BG}(n, c)$ to $\operatorname{BG}(\lambda n, \lambda c)$. She cuts the circle into $n$ intervals with $\lambda$ buckets, treats every interval as a super-bucket, and uses her strategy for $\mathrm{BG}(n, c)$ on the super-buckets. This yields $F(\lambda n, \lambda c) \leq F(n, c)$.

Lemma 2. (Monotonicity lemma) $F\left(n_{1}, c_{1}\right) \leq F\left(n_{2}, c_{2}\right)$ whenever $n_{1} / c_{1} \leq$ $n_{2} / c_{2}$.

Proof. This follows from $F\left(n_{1}, c_{1}\right)=F\left(n_{1} n_{2}, c_{1} n_{2}\right) \leq F\left(n_{1} n_{2}, c_{2} n_{1}\right)=$ $F\left(n_{2}, c_{2}\right)$.

By the definition of $F^{\prime}(n, c)$, Cinderella wins the game $\operatorname{BG}(n, c)$ if the bucket size is strictly larger than $F^{\prime}(n, c)$, and the Stepmother wins the game if the bucket size is strictly smaller than $F^{\prime}(n, c)$. What happens at the threshold $F^{\prime}(n, c)$ ?

Lemma 3. If $B G(n, c)$ is played with buckets of size $F^{\prime}(n, c)$, then Cinderella can keep the game running forever. 
Proof. Let $G(n, c, R)$ denote the maximum amount of water that the Stepmother can accumulate in some bucket within the first $R$ rounds of game $\mathrm{BG}(n, c)$; it can be proved by an inductive argument that this maximum indeed exists.

Now suppose that the Stepmother could enforce an overflow for buckets of size $F^{\prime}(n, c)$. Then she can enforce this overflow after a finite number $R$ of rounds, which means $G(n, c, R)>F^{\prime}(n, c)$. But then within $R$ rounds the Stepmother could as well enforce overflows for any bucket size between $F^{\prime}(n, c)$ and $G(n, c, R)$, which conflicts with the definition of $F^{\prime}(n, c)$.

Lower Bounds from Balancing Stepmothers. Our lower bound arguments for $F(n, c)$ use a special adversary which we call balancing Stepmother. A balancing Stepmother balances the water levels in certain buckets, and works in two phases. During the first phase, the Stepmother always distributes her liter in such a way that all $n$ buckets are filled to the same level. This common filling level is $1 / n$ in the first round, and in later rounds increases and converges to $1 / c$. The first phase ends, when the common filling level exceeds $1 / c-\varepsilon$ (where $\varepsilon$ is a tiny positive real number that can be made arbitrarily close to 0 ). The set of $n-c$ buckets that are filled to level $L_{1} \approx 1 / c$ at the end of the last round of the first phase is denoted by $S_{1}$.

In the second phase, we will usually ignore the dependence of our bounds on $\varepsilon$, so that the presentation remains simple and our formulas stay clean. The second phase goes through $n-c-1$ further rounds. At the beginning of the $r$-th one of these rounds $(r=1, \ldots, n-c-1)$, there are $(n-c)-r+1$ buckets filled to the same level $L_{r}$ that Cinderella could not empty in the preceding round; these buckets form the set $S_{r}$. The balancing Stepmother then picks an appropriate set $T_{r} \supseteq S_{r}$ of buckets, such that in the current round Cinderella must leave at least $(n-c)-r$ buckets in $T_{r}$ untouched. All buckets in $T_{r}$ are then filled to the same level $L_{r+1}=\left(\left|S_{r}\right| L_{r}+1\right) /\left|T_{r}\right|$. At the end of the last round $n-c-1$, there remains a single non-empty bucket whose contents $L_{n-c-1}$ forms the resulting lower bound.

Upper Bounds from Invariants. Our upper bound arguments for $F(n, c)$ are based on appropriate systems of invariants that (i) can be maintained by Cinderella, and that (ii) imply that every bucket contents remains below $F(n, c)$. A typical invariant system bounds the contents of every bucket by $x_{i}<F(n, c)$, and furthermore bounds the overall contents of certain groups of buckets. All invariants are trivially satisfied at the beginning of the first round when all buckets are empty. In our proofs we usually assume inductively that these invariants are satisfied at the beginning of some fixed round (just before the Stepmother moves), and then show that Cinderella can re-establish them at the end of the round. In doing this, we always let $x_{i}$ denote the contents of bucket $i$ at the beginning of the round, and we always let $y_{i}$ denote the contents of bucket $i$ after the Stepmother has moved. 


\section{Above the Half-Diagonal}

By definition the games $\mathrm{BG}(n, c)$ above the half-diagonal satisfy $n<2 c$. It is not hard to see that all these games satisfy $F(n, c)<1$ (as at the end of her move, Cinderella can keep the total amount of water in the system below $n / c-1)$. We fully understand the games $\mathrm{BG}(c+1, c), \mathrm{BG}(c+2, c)$, and $\mathrm{BG}(c+3, c)$.

Theorem 1. $F(c+1, c)=1 / c$ holds for all $c \geq 1$.

Proof. (Upper bound) As invariant, Cinderella always leaves a single bucket untouched whose contents is below 1/c. The Stepmother adds one liter to the system and increases the total amount of water to less than $(c+1) / c$. By averaging, one of the $c+1$ buckets has contents below $1 / c$, and that's the bucket that Cinderella does not touch in her move.

(Lower bound) In her first phase, the balancing Stepmother brings the contents of all buckets arbitrarily close to $1 / c$.

Theorem 2. $F(c+2, c)=(2 c+4) /\left(c^{2}+3 c\right)$ holds for all odd $c \geq 1$, and $F(c+2, c)=2 / c$ holds for all even $c \geq 2$.

Theorem 3. The values $F(c+3, c)$ behave as follows for $c \geq 1$.

(i) $F(3 s+2,3 s-1)=1 / s$

(ii) $F(3 s+3,3 s)=1 / s$

(iii) $F(3 s+4,3 s+1)=\frac{(s+1)(6 s+11)}{(s+2)(2 s+3)(3 s+1)}$

Theorem 4. $F(9,5)=299 / 525$.

The proofs of Theorems 2, 3 and 4 are to be found in the subsections below. It is easily verified that these theorems (together with monotonicity) imply all entries for $n \leq 10$ above the half-diagonal of Table 1 .

We think that also all values $F(c+4, c)$ are within reach and could be fully characterized, if one invests sufficient time and energy. We actually determined many values $F(n, c)$ above the half-diagonal with the help of computer programs. For instance we know that $F(13,9)=37 / 105$ and $F(17,13)=1961 / 7605$, and it took us hours of computation time to establish $F(16,11)=252 / 715$. We see many patterns and regularities in the data, but we can not find a unifying conjecture that would systematically cover all possible cases; certain divisibility properties seem to kick in and totally mess up the structure. Our data suggests the following conjecture (which is fairly weak and only covers a small part of the unknown area).

Conjecture 1. $F(n, c)=1 / 2$ holds for all $n$ and $c$ with $3 / 2 \leq n / c<5 / 3$.

\subsection{The Proof of Theorem 2}

Since monotonicity settles the cases with even $c$, we only discuss the games where $c$ is odd (and $n=c+2$ ). 
(Upper bound) At the end of every round, Cinderella leaves two non-empty buckets (say buckets 1 and 2) whose loads $x_{1}$ and $x_{2}$ satisfy the following two invariants:

$$
\begin{aligned}
& x(1,2)<2 / c \\
& x_{1}, x_{2}<L_{2}:=(2 c+4) /\left(c^{2}+3 c\right)
\end{aligned}
$$

Then the Stepmother moves and yields bucket contents $y_{1}, \ldots, y_{c+2}$. Cinderella maintains the invariants by leaving a pair $j, j+1$ of neighboring buckets with smallest total contents. Since the Stepmother only adds a single liter, invariant (1a) implies

$$
\sum_{i=1}^{c+2} y_{i}<(c+2) / c .
$$

By averaging we get $y(j, j+1) \leq\left(2 \sum y_{i}\right) /(c+2)<2 / c$, which ensures invariant (1a). Next, suppose for the sake of contradiction that $y_{j} \geq L_{2}$. Partition the remaining $c+1$ buckets (except bucket $j$ ) into $(c+1) / 2$ pairs of neighboring buckets. The total contents of every such pair is at least $y(j, j+1) \geq L_{2}$, which implies $\sum_{i=1}^{c+2} y_{i} \geq \frac{1}{2}(c+3) L_{2}=(c+2) / c$, and thus contradicts (2). Hence $y_{j}<L_{2}$, and an analogous argument yields $y_{j+1}<L_{2}$.

(Lower bound) The first phase of the balancing Stepmother ends with two buckets (say 1 and 2) of contents very close to $1 / c$. In the second phase, the Stepmother chooses set $T_{1}$ to contain buckets 1 and 2 together with all buckets with even numbers; note that $\left|T_{1}\right|=(c+3) / 2$. Then all buckets in $T_{1}$ are brought to level at least $(2 / c+1) /\left|T_{1}\right|=L_{2}$. Since Cinderella cannot simultaneously empty all buckets in $T_{1}$, we get $F(c+2, c) \geq L_{2}$.

\subsection{The Proof of Theorem $[3$. (i) and (ii)}

Monotonicity and Theorem 1 yield the lower bound $F(3 s+2,3 s-1) \geq F(3 s+$ $3,3 s)=F(s+1, s)=1 / s$. Hence we will concentrate on the upper bound for the game with $n=3 s+2$ buckets and $c=3 s-1$.

At the end of some fixed round Cinderella leaves three adjacent buckets, say the buckets $3,4,5$. She always maintains the following two invariants.

$$
\begin{aligned}
x_{4} & <1 / s \\
x(3,5) & <1 / s
\end{aligned}
$$

The Stepmother adds one liter to the system and brings the contents to $y_{1}, y_{2}, \ldots, y_{3 s+2}$. A triple is a group of three consecutive buckets $i, i+1, i+2$ in the circle. A triple is called good, if $y(i, i+1, i+2)<1 / s$. By emptying all buckets outside a good triple, Cinderella can maintain the invariants. Hence we assume from now on that there is no good triple.

We denote by $W$ the total amount of water in all buckets except bucket 4 . Invariant $(3 \mathrm{~b})$ implies $W<1+1 / s$. Since there are no good triples, we have 
$y(1,2,3) \geq 1 / s$ and $y(5,6,7) \geq 1 / s$. By subtracting these two inequalities from $W<1+1 / s$, we get

$$
\sum_{i=8}^{3 s+2} y_{i}<1-1 / s .
$$

Next suppose for the sake of contradiction that $y_{3 i+2} \geq 1 / s$ holds for some $i$ with $2 \leq i \leq s$. Then the $3 i-6$ buckets $8,9, \ldots, 3 i+1$ and the $3 s-3 i$ buckets $3 i+3,3 i+4, \ldots, 3 s+2$ can be divided into $s-2$ non-good triples. Therefore the overall amount of water in these $s-2$ triples together with $y_{3 i+2}$ would be at least $(s-1) / s$, which contradicts (4). This contradiction implies $y_{3 i+2}<1 / s$ for $2 \leq i \leq s$. Furthermore we assume $y(3 i+1,3 i+3) \geq 1 / s$, since otherwise Cinderella could easily maintain the invariants by emptying all buckets except the triple $3 i+1,3 i+2,3 i+3$. Summing these $s-1$ inequalities for $2 \leq i \leq s$ yields

$$
\sum_{i=2}^{s} y_{3 i+1}+\sum_{i=2}^{s} y_{3 i+3} \geq 1-1 / s
$$

If $y(6,8) \geq 1 / s$, then (5) yields that the Stepmother has added her entire liter to the buckets outside the triple 3,4,5, and Cinderella can maintain all invariants by reverting the system to the preceding state. Hence we assume from now on $y(6,8)<1 / s$, and a symmetric argument yields $y(3 s+2,2)<1 / s$. If $y_{7}<1 / s$ or $y_{1}<1 / s$, then Cinderella maintains the invariants by emptying everything except the triple $6,7,8$, respectively by emptying everything except the triple $3 s+2,1,2$. Hence we assume from now $y_{7} \geq 1 / s$ and $y_{1} \geq 1 / s$.

Finally note that the $3 s-5$ buckets $8,9,10, \ldots, 3 s+2$ contain $s-2$ pairwise disjoint triples, each of which is non-good and has total contents at least $1 / \mathrm{s}$. Together with $y_{1} \geq 1 / s$ and $y_{7} \geq 1 / s$ this shows that the Stepmother must have added her entire liter to the buckets $7,8,9,10, \ldots, 3 s+2$, and 1. By emptying these buckets, Cinderella reverts the system to the preceding state and maintains all invariants.

\subsection{The Proof of Theorem 3 . (iii)}

We discuss the game with $n=3 s+4$ and $c=3 s+1$. For $s \geq 1$, we introduce three parameters $L_{1}, L_{2}, L_{3}$ by

$$
\begin{aligned}
& L_{1}=\frac{1}{3 s+1} \\
& L_{2}=\frac{3 s+4}{(2 s+3)(3 s+1)} \\
& L_{3}=\frac{(s+1)(6 s+11)}{(s+2)(2 s+3)(3 s+1)}
\end{aligned}
$$


Note that these three parameters satisfy

$$
L_{2}=\frac{3 L_{1}+1}{2 s+3} \quad \text { and } \quad L_{3}=\frac{2 L_{2}+1}{s+2} .
$$

Furthermore, we have

$$
L_{1} \leq L_{2} \leq L_{3} \leq 2 L_{2} \leq 3 L_{1}
$$

and finally there is the useful inequality

$$
3 L_{1}+1 \leq(s+1) L_{3}+2 L_{2} .
$$

(Upper bound) At the end of some fixed round Cinderella leaves three non-empty buckets, say buckets $1,2,3$. She maintains the following three invariants.

$$
\begin{aligned}
x_{i} & <L_{3} & & \text { for } 1 \leq i \leq 3 \\
x(i, j) & <2 L_{2} & & \text { for } 1 \leq i<j \leq 3 \\
x(1,2,3) & <3 L_{1} & &
\end{aligned}
$$

Then the Stepmother moves, and raises the bucket contents to $y_{1}, y_{2}, \ldots, y_{3 s+4}$. By invariant (9c) the overall amount of water in the system is bounded by

$$
\sum_{j=1}^{3 s+4} y_{j}<3 L_{1}+1
$$

A bucket $i$ with $y_{i} \geq L_{3}$ is called large. A triple is a group of three consecutive buckets $i, i+1, i+2$ in the circle. A triple is called good, if (i) none of its buckets is large and (ii) $y(i, i+1, i+2)<2 L_{2}$. If Cinderella empties all buckets outside a good triple, she automatically maintains the invariants. Hence we assume from now on that there is no good triple.

Lemma 4. If there is no good triple, then there also are no large buckets.

Proof. We distinguish several cases on the number $\ell$ of large buckets. The overall amount of water in the system is at least $\ell L_{3}$, and below $3 L_{1}+1$ by (10). By using (8) and (7) this yields

$$
\ell L_{3}<3 L_{1}+1 \leq(s+1) L_{3}+2 L_{2} \leq(s+3) L_{3} .
$$

Therefore $\ell \leq s+2$. If $\ell=s+2$ and at most two of the buckets $1,2,3$ are large, then (9b) implies that the overall amount $W$ of water in the large buckets satisfies

$$
(s+2) L_{3} \leq W<2 L_{2}+1,
$$

which contradicts (6). If $\ell=s+2$ and all three buckets $1,2,3$ are large, then these $s+2$ large buckets divide the $2 s+2$ non-large buckets into at most $s$ nonempty intervals along the circle. One of these intervals contains at least three 
non-large buckets, and hence a non-good triple whose total contents is at least $2 L_{2}$. Then the overall amount $W$ of water in the large buckets plus the water in this non-good triple satisfies

$$
(s+2) L_{3}+2 L_{2} \leq W<3 L_{1}+1,
$$

which contradicts (8). In the remaining cases we have $\ell \leq s+1$.

If $\ell \geq 1$, the large buckets divide the $3 s+4-\ell$ non-large buckets into $\ell$ intervals along the circle. If an interval consists of $k$ non-large buckets, we can find $\lfloor k / 3\rfloor$ pairwise disjoint triples in this interval. It can be seen that altogether we find at least $s+2-\ell$ pairwise disjoint triples in all $\ell$ intervals. Each of these triples is non-good and has total contents at least $2 L_{2}$. By applying (10) the total contents $W$ of all buckets satisfies

$$
\ell L_{3}+2 L_{2}(s+2-\ell) \leq W<3 L_{1}+1
$$

Since $L_{3}-2 L_{2} \leq 0$, the expression in the left hand side is decreasing in $\ell$. Together with $\ell \leq s+1$ this yields $(s+1) L_{3}+2 L_{2}<3 L_{1}+1$, which contradicts (8). This leaves $\ell=0$ as the only possible case.

By the lemma there is no large bucket, and we see that all buckets a priori satisfy invariant (9a). Consider a fixed bucket $i$, and divide the remaining $3 s+3$ buckets into $s+1$ non-good triples. Then

$$
y_{i}+(s+1) \cdot 2 L_{2} \leq \sum_{j=1}^{3 s+4} y_{j}<3 L_{1}+1=(2 s+3) L_{2}
$$

which implies $y_{i}<L_{2}$. Hence any pair of buckets satisfies invariant (9b). By averaging, there exists a triple of buckets whose total contents is

$$
y_{j}+y_{j+1}+y_{j+2}<3 \cdot \frac{3 L_{1}+1}{3 s+4}=3 L_{1} \text {. }
$$

Cinderella empties all buckets except this triple, and thereby also fulfills invariant (9c).

(Lower bound) The first phase of the balancing Stepmother ends with three buckets (say buckets $1,2,3$ ) having contents very close to $L_{1}$. The second phase goes through two further rounds.

In the first of these rounds, the Stepmother selects the set $T_{1}$ to contain all buckets except the buckets $3 i+1$ with $i=1, \ldots, s+1$. Then $T_{1}$ contains $2 s+3$ buckets which the Stepmother all brings to contents $L_{2}$. Cinderella leaves a set $S_{2}$ of two buckets with contents $L_{2}$; these two buckets are either adjacent (say 1 and 2) or separated by a single other bucket (say buckets $3 s+4$ and 2).

In the second round, the Stepmother selects the set $T_{2}$ to contain the two buckets in set $S_{2}$ together with the buckets $3 i+2$ with $i=1, \ldots, s$. Then $T_{2}$ consists of $s+2$ buckets which the Stepmother all brings to contents $L_{3}$. Cinderella must leave one bucket with contents $L_{3}$ at the end of the round. 


\section{The Proof of Theorem 4}

(Upper bound) Assume that in the game BG(9,5), Cinderella leaves a bucket configuration that satisfies the following four invariants.

$$
\begin{aligned}
x_{i}<299 / 525 & \approx 0.569 & & \text { for } 1 \leq i \leq 9 \\
x(S)<124 / 175 & \approx 0.708 & & \text { for all } S \text { with }|S|=2 \\
x(S)<27 / 35 & \approx 0.771 & & \text { for all } S \text { with }|S|=3 \\
x(S)<\quad 4 / 5 & =0.800 & & \text { for all } S \text { with }|S|=4
\end{aligned}
$$

The Stepmother moves and raises the bucket contents from $x_{1}, \ldots, x_{9}$ to $y_{1}, \ldots, y_{9}$. Note that $\sum_{i=1}^{9} y_{i}<9 / 5$ by (11d). A quadruple is a set of four consecutive buckets in the circle. A quadruple is called good, if its four buckets satisfy (11a)-111d). If there is a good quadruple, then Cinderella can maintain the invariants by emptying all buckets outside the quadruple.

Lemma 5. If $y_{j} \geq 299 / 525$ for some $j$, then Cinderella can maintain the invariants.

Proof. A bucket $j$ with $y_{j} \geq 299 / 525=: L$ is called large. If the Stepmother leaves three large buckets $i, j, k$, then $x(i, j, k) \geq y(i, j, k)-1 \geq 3 L-1=$ 124/175. This implies that $x_{i}, x_{j}, x_{k}$ all are non-zero, since otherwise two of these buckets would have violated (11b). Hence $i, j, k$ all belong to the quadruple that Cinderella did not touch in the preceding round. If Cinderella empties this quadruple (together with some fifth bucket), the remaining volume of water decreases to $9 / 5-3 L<124 / 175$, and all invariants are maintained.

If the Stepmother leaves two large buckets $i$ and $j$, then Cinderella empties these large buckets (together with three other buckets). The remaining volume of water decreases to $9 / 5-2 L<124 / 175$, and all invariants are maintained.

Finally assume that the Stepmother leaves a single large bucket, which without loss of generality is bucket 1 . Then $y(2,3,4,5)+y(6,7,8,9) \leq 9 / 5-L<2$. $124 / 175$, which implies that one of the quadruples $2,3,4,5$ and $6,7,8,9$ must be good.

Lemma 6. If $y_{j} \geq 62 / 175$ for some $j$, then Cinderella can maintain the invariants.

Proof. By the preceding lemma we assume $y_{i}<299 / 525$ for all $i$. We assume furthermore that bucket 1 with $y_{1} \geq 62 / 175$ is the fullest bucket, and that the quadruples $2,3,4,5$ and $6,7,8,9$ both are non-good (so that the total contents of either quadruple is at least 124/175). If the quadruple $2,3,4,5$ violates (11C) or (11d), then we would get the contradiction

$$
y_{1}+y(2,3,4,5)+y(6,7,8,9) \geq 62 / 175+27 / 35+124 / 175>9 / 5 .
$$

Hence the quadruple $2,3,4,5$ contains two buckets $b_{1}, b_{2}$ that violate (11b) with $y\left(b_{1}, b_{2}\right) \geq 124 / 175$. Symmetric arguments show that the quadruple $6,7,8,9$ contains two buckets $b_{3}, b_{4}$ with $y\left(b_{3}, b_{4}\right) \geq 124 / 175$. 
Let $T=\left\{1, b_{1}, b_{2}, b_{3}, b_{4}\right\}$ and note $x(T) \geq y(T)-1 \geq 27 / 35$. Now (11c) implies that $T$ contains all the four buckets that Cinderella did not touch in the preceding round. By emptying this quadruple (together with some fifth bucket), the remaining volume of water goes below 124/175 and all invariants are maintained.

By the above lemmas we assume from now on $y_{i}<62 / 175$ for all $i$, so that invariants (11a) and (11b) become harmless. Consider an arbitrary bucket $k$, and consider the partition of the remaining eight buckets into two quadruples $T_{1}$ and $T_{2}$, so that

$$
y_{k}<9 / 5-y\left(T_{1}\right)-y\left(T_{2}\right) .
$$

We may assume that both quadruples $T_{1}$ and $T_{2}$ are non-good. Then the lower bounds $y\left(T_{1}\right), y\left(T_{2}\right) \geq 27 / 35$ and (12) together yield $y_{k}<9 / 35$. Since $k$ was an arbitrary bucket, this means that every bucket triple satisfies (11c), which also makes invariant (11c) harmless. Since $T_{1}$ and $T_{2}$ are non-good, we now conclude $y\left(T_{1}\right), y\left(T_{2}\right) \geq 4 / 5$. But then (12) yields $y_{k}<1 / 5$ for all $k$, and any move of Cinderella will maintain all invariants. This completes the proof.

(Lower bound) The first phase of the balancing Stepmother ends with four consecutive buckets (say buckets $1,2,3,4$ ) having contents very close to $1 / 5$. The second phase goes through three further rounds.

In the first of these rounds, the Stepmother uses set $T_{1}=\{1,2,3,4,6,7,8\}$ with all buckets except 5 and 9 . The Stepmother brings every bucket in $T_{1}$ to contents $9 / 35$. Cinderella leaves a set of four buckets, at least three of which are in $T_{1}$. These three buckets are either adjacent (say 2,3,4 in this first case) or separated by a single empty bucket (say $3,4,6$ in the second case).

In the second round the Stepmother selects the set $T_{2}$ to contain five buckets; in the first case she uses $T_{2}=\{2,3,4,7,8\}$ and in the second case $T_{2}=\{3,4,6,7,8\}$. The Stepmother brings every bucket in $T_{2}$ to contents $62 / 175$. Cinderella leaves a set of four buckets, at least two of which are in $T_{2}$. We rename the buckets so that 1 and $b \in\{2,3,4\}$ keep their contents $62 / 175$.

In the third round the Stepmother uses $T_{3}=\{1, b, 6\}$, and fills these three buckets up to level 299/525. Cinderella must leave at least one such bucket with contents $299 / 525$ at the end of the round.

\section{Below the Half-Diagonal}

By definition the games $\operatorname{BG}(n, c)$ below the half-diagonal satisfy $n \geq 2 c$. For these games the harmonic sums $H_{k}=1+\frac{1}{2}+\frac{1}{3}+\frac{1}{4}+\ldots+\frac{1}{k}$ seem to play a major role. The following theorem has been observed before by Dietz \& Sleator 4] and Chrobak \& al. 3].

Theorem 5. $F(n, 1)=H_{n-1}$ holds for all $n \geq 2$.

Proof. (Upper bound) Let $x_{i}$ denote the contents of bucket $i$ at the beginning of some round. We argue that Cinderella can maintain the following invariants.

$$
x(T)<\left(1+H_{n-1}-H_{|T|}\right)|T| \quad \text { for all bucket sets } T
$$


The Stepmother raises the bucket contents to $y_{1}, \ldots, y_{n}$, and we assume that $y_{n} \geq y_{i}$ for all $i$. Then for any bucket set $T \subseteq\{1, \ldots, n-1\}$ we have

$$
\begin{aligned}
\frac{1}{|T|} y(T) & \leq \frac{1}{|T|+1}\left(y(T)+y_{n}\right) \leq \frac{1}{|T|+1}\left(x(T)+x_{n}+1\right) \\
& <1+H_{n-1}-H_{|T|+1}+\frac{1}{|T|+1}=1+H_{n-1}-H_{|T|} .
\end{aligned}
$$

Therefore Cinderella can maintain the invariants by emptying the fullest bucket $n$. By applying (13) to a single bucket set $T=\{i\}$, we get that all buckets satisfy $x_{i}<H_{n-1}$.

(Lower bound) In the first phase, the balancing Stepmother brings the filling level of all buckets very close to 1 . The first phase terminates with a set $S_{1}$ of $n-1$ buckets with contents $L_{1} \approx 1$. In the second phase, the Stepmother always chooses $T_{r}:=S_{r}$ as the set of the $n-r$ currently fullest buckets (which Cinderella could not empty in the preceding round), and fills all of them to level $L_{r+1}=1+H_{n-1}-H_{n-r-1}$. Then at the end of round $n-2$ Cinderella has left a bucket of contents $H_{n-1}$.

Theorem 6. (i) $F(7,2)=3 / 2$ and (ii) $F(9,2)=11 / 6$.

Theorem 7. $F(n, c)=1$ holds for all $n$ and $c$ with $2 \leq n / c<3$.

The proof of Theorem 6 and the (long and technical) proof of Theorem 7 can be found in the full version of this paper. Note that the theorems in this section together with the monotonicity property imply all entries for $n \leq 10$ below the half-diagonal of Table 1. Furthermore Theorem[7 covers the cases with $\lfloor n / c\rfloor=2$ for the following clean and natural conjecture.

Conjecture 2. $F(n, c)=F(\lfloor n / c\rfloor, 1)$ holds for all $n$ and $c$ with $2 \leq n / c$.

If true, then this conjecture (in combination with Theorem 5 ) would determine all values of $F(n, c)$ below the half-diagonal. Note that the monotonicity Lemma 2 yields $F(n, c) \geq F(\lfloor n / c\rfloor, 1)$, and that therefore the hard part of the conjecture is to come up with the right systems of invariants. Unfortunately, we have no idea how to settle Conjecture 2, In fact, we cannot even settle the special case $F(13,2)=137 / 60$. The games $\mathrm{BG}(n, 2)$ with odd $n \leq 11$ can be handled by certain types of invariant systems that we understand very well; all these systems are built around subsets of pairwise non-adjacent buckets that follow a certain pattern. With the help of YICES we can prove that the most natural generalization of this pattern to $\operatorname{BG}(13,2)$ will not work out, since there exist situations where Cinderella cannot maintain the corresponding invariants.

\section{Final Remarks}

We have settled all bucket games $\operatorname{BG}(n, c)$ with $n \leq 12$. Some of our smaller results started to grow together, and eventually resulted in general theorems 
that cover large families of games (as for instance the families in Theorem 3 and Theorem 7). There remain many open questions, and in particular there remains our tantalizing Conjecture 2 .

All our lower bounds have been derived by a suitable balancing Stepmother strategy (sometimes in combination with monotonicity). For many games, we performed extensive computer experiments and used backtracking algorithms (written in Haskell) to detect the strongest balancing Stepmothers; this boils down to checking a huge but finite number of cases.

Question 1. Does every value $F(n, c)$ result from an adversary argument with a balancing Stepmother (in combination with monotonicity)?

A positive answer to Question 1 would also imply the truth of the following conjecture.

Conjecture 3. The function $F(n, c)$ only takes rational values, and is Turingcomputable.

For some of the considered games it was far from clear how to choose the right system of invariants, and several attempts were required before finding the right choice. For experimenting with such invariants it was convenient to use an SMT solver (Satisfiability Modulo Theories) which checks the satisfiability of any Boolean formula on linear inequalities. Note that this goes far beyond linear programming, as in linear programming the set of constraints is the conjunction of a set of linear inequalities, whereas in SMT any combination of disjunctions and conjunctions is allowed. Now $I$ is an invariant system for Cinderella if the formula

$$
I \wedge\left(\sum_{i} y_{i}=1+\sum_{i} x_{i}\right) \wedge\left(\bigwedge_{i} y_{i} \geq x_{i}\right) \wedge \bigvee_{j} \neg I_{j}
$$

is unsatisfiable. Here the formula $I_{j}(1 \leq j \leq n)$ is obtained from $I$ as follows. For $i=j+1, \ldots, j+c$ every occurrence of $x_{i}$ is replaced by 0 , and for the remaining indices $i$ every occurrence of $x_{i}$ is replaced by $y_{i}$. Our approach was to check this by the SMT solver YICES for several candidates for $I$. If this formula is unsatisfiable, we have proved the invariance and thereby derived an upper bound on $F(n, c)$; if it is satisfiable then YICES provides the corresponding values of $x_{i}$ and $y_{i}$ that can be interpreted as a counterexample for the invariance. Internally, YICES works with rational numbers in unbounded precision, and typically the proof trees consist of thousands of indigestible case distinctions, but are found within at most a few seconds.

\section{References}

1. Bender, M.A., Fekete, S.P., Kröller, A., Mitchell, J.S.B., Liberatore, V., Polishchuk, V., Suomela, J.: The minimum-backlog problem. In: Proceedings of the International Conference on Mathematical Aspects of Computer and Information Sciences (MACIS 2007), pp. 1-18 (2007) 
2. Bodlaender, M.H.L., Hurkens, C.A.J., Woeginger, G.J.: The Cinderella Game on Holes and Anti-holes. In: Kolman, P., Kratochvíl, J. (eds.) WG 2011. LNCS, vol. 6986, pp. 71-82. Springer, Heidelberg (2011)

3. Chrobak, M., Csirik, J.A., Imreh, C., Noga, J., Sgall, J., Woeginger, G.J.: The Buffer Minimization Problem for Multiprocessor Scheduling with Conflicts. In: Yu, Y., Spirakis, P.G., van Leeuwen, J. (eds.) ICALP 2001. LNCS, vol. 2076, pp. 862874. Springer, Heidelberg (2001)

4. Dietz, P.F., Sleator, D.D.: Two algorithms for maintaining order in a list. In: Proceedings of the 19th Annual ACM Symposium on Theory of Computing (STOC 1987), pp. 365-372 (1987)

5. Dutertre, B., de Moura, L.: Yices - A high-performance SMT solver. Downloadable from http://yices.csl.sri.com

6. Hurkens, A.J.C., Hurkens, C.A.J., Woeginger, G.J.: How Cinderella won the bucket game (and lived happily ever after). Mathematics Magazine 84, 285-290 (2011)

7. Polishchuk, V., Suomela, J.: Optimal Backlog in the Plane. In: Fekete, S. (ed.) ALGOSENSORS 2008. LNCS, vol. 5389, pp. 141-150. Springer, Heidelberg (2008)

8. Woeginger, G.J.: Combinatorics problem C5. In: Problem Shortlist of the 50th International Mathematical Olympiad, Bremen, Germany, pp. 33-35 (2009) 